\title{
CONSTRUCTION OF STEINER QUADRUPLE SYSTEMS HAVING LARGE NUMBERS OF NONISOMOR PHIC ASSOCIATED STEINER TRIPLE SYSTEMS
}

\author{
CHARLES C. LINDNER ${ }^{1}$
}

\begin{abstract}
If $(Q, q)$ is a Steiner quadruple system and $x$ is any element in $Q$ it is well known that the set $Q_{x}=Q \backslash\{x\}$ equipped with the collection $q(x)$ of all triples $\{a, b, c\}$ such that $\{a, b, c, x\} \in q$ is a Steiner triple system. A quadruple system $(Q, q)$ is said to have at least $n$ nonisomorphic associated triple systems (NATS) provided that for at least one subset $X$ of $Q$ containing $n$ elements the triple systems $\left(Q_{x}, q(x)\right)$ and $\left(Q_{y}, q(y)\right)$ are nonisomorphic whenever $x \neq y \in X$. Prior to the results in this paper the maximum number of known NATS for any quadruple system was 2 . The main result in this paper is the construction for each positive integer $t$ of a quadruple system having at least $t$ NATS.
\end{abstract}

1. Introduction. A Steiner quadruple system (or more simply a quadruple system) is a pair $(Q, q)$ where $Q$ is a finite set and $q$ is a collection of 4 element subsets of $Q$ (called blocks) such that any three distinct elements of $Q$ belong to exactly one block of $q$. The number $|\mathrm{Q}|$ is called the order of the quadruple system $(Q, q)$. Hanani proved in 1960 that the spectrum for quadruple systems consisted of the set of all positive integers $n \equiv 2$ or 4 $(\bmod 6)[3]$. It is easy to show that a quadruple system of order $n$ has $n(n-1)(n-2) / 24$ blocks. If $(Q, q)$ is a quadruple system and $x$ is any element in $Q$ we will denote $Q \backslash\{x\}$ by $Q_{x}$ and the set of all triples $\{a, b, c\}$. such that $\{x, a, b, c\} \in q$ by $q(x)$. It is a routine matter to see that $\left(Q_{x}, q(x)\right)$ is a Steiner triple system and is called an associated triple system (of the quadruple system $(Q, q)$ ). A very interesting problem is the construction of quadruple systems $(Q, q)$ such that for some subset $X$ of $Q$ containing at least two elements, the associated Steiner triple systems $\left(Q_{x}, q(x)\right)$ and $\left(Q_{y}, q(y)\right)$ are nonisomorphic whenever $x \neq y \in X$. If $|X|=n$ we will say that $(Q, q)$ has at least $n$ nonisomorphic associated triple systems (NATS).

Received by the editors January 13, 1974 .

AMS (MOS) subject classifications (1970). Primary 05B05; Secondary 62K10.

Key words and phrases. Steiner quadruple system, Steiner triple sy stem, nonisomorphic associated triple systems.

${ }^{1}$ Research supported by National Science Foundation Grant GP-37629. 
In [6], N. S. Mendelsohn and H. S. Y. Hung have shown that there are exactly four nonisomorphic quadruple systems of order 14. Two of these quadruple systems have 2 NATS. In [4] the author used one of the se quadruple systems and the ordinary direct product to construct an infinite class of quadruple systems having at least 2 NATS. To date the results in [4] and [6] are the only known results on the number of NATS of quadruple systems. In all cases the maximum number of NATS obtained is 2, so that to date there are no known quadruple systems having more than 2 NATS. In this paper we rectify this situation by giving a construction for quadruple systems which yields for any positive integer $t$ a quadruple system having at least $t$ NATS. The construction used was first introduced by the author in [5] in order to construct nonisomorphic quadruple systems. One interesting feature of this construction is that it is nonrecursive insofar as it does not depend on quadruple systems of smaller order having large numbers of NATS.

2. Construction of Steiner quadruple systems. By a 3-skein is meant a pair $(Q,\langle,\rangle$,$) where Q$ is a finite set and $\langle,$,$\rangle is a temary operation on$ $Q$ such that if in the equation $\langle x, y, x\rangle=w$ any three of $x, y, z$, and $w$ are given then the remaining element is uniquely determined.

Let $(Q, b(q))$ and $(V, b(v))$ be quadruple systems based on $Q=\{1,2$, $\cdots, q\}$ and $V=\{1,2, \cdots, v\}$ respectively and let $(Q,\langle\rangle$,$) be a 3$-skein. Define on the set $Q \times V$ the following collection $b$ of 4 element subsets.

(1) For every block $\{a, b, c, d\} \in b(q)$ and every $w \in V$,

$$
\{(a, w),(b, w),(c, w),(d, w)\} \in b .
$$

(2) For every two element subset $\{a, b\}$ of $Q$ and every two element subset $\{u, w\}$ of $V$,

$$
\{(a, u),(b, u),(a, w),(b, w)\} \in b .
$$

(3) For every block $\{a, b, c, d\} \in b(q)$ and every two element subset $\{u, w\}$ of $V$ the 6 subsets listed below belong to $b$ :

$$
\begin{array}{ll}
\{(a, u),(b, u),(c, w),(d, w)\} & \{(a, w),(b, u),(c, u),(d, w)\} \\
\{(a, u),(b, w),(c, u),(d, w)\} & \{(a, w),(b, u),(c, w),(d, u)\} \\
\{(a, u),(b, w),(c, w),(d, u)\} & \{(a, w),(b, w),(c, u),(d, u)\}
\end{array}
$$

(4) For every block $\{x, y, z, w\} \in b(v)$ and every three (not necessarily distinct) elements $p, q$, and $s$ of $Q$,

$$
\{(p, x),(q, y),(s, z),(\langle p, q, s\rangle, w)\} \in b \quad \text { where } x<y<z<w .
$$


Theorem $1[5] .(Q \times V, b)$ is a Steiner quadruple system.

We now modify the construction of $(Q \times V, b)$ by leaving blocks of types (1), (2), and (3) unchanged and by replacing blocks of type (4) as follows.

Let

$$
\{x, y, z, w\}_{1},\{x, y, z, w\}_{2}, \cdots,\{x, y, z, w\}_{t}
$$

be the $t=v(v-1)(v-2) / 24$ blocks of $b(v)$ and let

$$
\left(Q,\langle,,\rangle_{1}\right),\left(Q,\langle,,\rangle_{2}\right), \cdots,\left(Q,\langle,,\rangle_{t}\right)
$$

be any $t=v(v-1)(v-2) / 24$ 3-skeins. For every block $\{x, y, z, w\}_{i}$ and every three elements $p, q, s \in Q$ insert the block

$$
\left\{(p, x),(q, y),(s, z),\left(\langle p, q, s\rangle_{i}, w\right)\right\}
$$

where $x<y<z<w$. Denote this new system by $\left(Q \times V, b^{*}\right)$.

Theorem $2[5] .\left(Q \times V, b^{*}\right)$ is a Steiner quadruple system.

We remark that the 3 -skeins $\left(Q,\langle,\rangle_{1}\right),\left(Q,\langle,,\rangle_{2}\right), \cdots,\left(Q,\langle,\rangle_{t}\right)$ are not necessarily related. This observation is crucial in what follows.

3. Construction of quadruple systems having a large number of NATS. Let $(Q,\langle,\rangle$,$) be any 3$-skein and let $x \in Q$. Define a binary operation $o(x)$ on $Q$ by $a d(x) b=\langle x, a, b\rangle$. Then $(Q, o(x))$ is a quasigroup. It will be convenient to denote $\alpha(x)$ by $\langle x,$,$\rangle in what follows. Conversely, any$ quasigroup $(Q, \odot)$ can be obtained from a 3 -skein in this manner by taking $a$ to be a cycle of length $|Q|$ on $Q$ and defining $\langle a, b, c\rangle=(b \odot c) a^{i}$, where $x \alpha^{i}=a$. Since there are at least $q !(q-1) ! \cdots 2 \cdot 1$ (the product of the first $q$ factorials) distinct quasigroups of order $q$ [1], for each $x \in Q$ there are at least $q !(q-1) ! \cdots 2 \cdot 1$ distinct quasigroups $(Q,\langle x,\rangle$,$) ,$ all 3-skeins $(Q,\langle,\rangle$,$) .$

Theorem 3. Let $q$ and $v, v>4$, be positive integers $\equiv 2$ or $4(\bmod 6)$ with

$$
(q !(q-1) ! \cdots 2 \cdot 1) /(q v-1) !>v .
$$

Then there is a Steiner quadruple system of order qu having at least $v$ NATS.

Proof. Let $(v, b(v))$ be a quadruple system of order $v$ and denote by $V(x)$ the set of blocks in $b(v)$ containing the element $x$. Since

$$
|V(x)|=(v-1)(v-2) / 6>v,
$$


the sets $V(1), V(2), \ldots, V(v)$ have a system of distinct representatives [2] which we will denote by

$$
\left\{1, x_{1}, y_{1}, z_{1}\right\},\left\{2, x_{2}, y_{2}, z_{2}\right\}, \cdots,\left\{v, x_{v}, y_{v}, z_{v}\right\}
$$

Now let $(Q, b(q))$ be any quadruple system of order $q$ and

$$
\left(Q,\langle,,\rangle_{1}\right),\left(2,\langle,,\rangle_{2}\right), \cdots,\left(Q,\langle,,\rangle_{t}\right)
$$

any $t=v(v-1)(v-2) / 243$-skeins. As previously mentioned, the 3-skeins $\left(Q,\langle,,\rangle_{i}\right), i=1,2, \cdots, t$, can be chosen independently of each other in constructing the quadruple system $\left(Q \times V, b^{*}\right)$ given in Theorem 2 . Hence if $\left(Q,\langle,\rangle_{i}\right), i=1,2, \cdots, v$, is always associated with the block $\left\{i, x_{i}, y_{i}, z_{i}\right\}$, then independently of any other considerations, by allowing $\left(Q,\langle,,\rangle_{i}\right)$ to run over all distinct 3 -skeins we can use Theorem 2 to construct at least $q !(q-1) ! \cdots 2 \cdot 1$ distinct quadruple systems of order $q v$ any two of which differ by at least one block of the form

$$
\left\{(1, i),\left(a, x_{i}\right),\left(b, y_{i}\right),\left(\langle 1, a, b\rangle_{i}, z_{i}\right)\right\} \text {. }
$$

Therefore the associated triple systems obtained from each of these quadruple systems by deleting $(1, i)$ differ by at least one triple of the form

$$
\left\{\left(a, x_{i}\right),\left(b, y_{i}\right),\left(\langle 1, a, b\rangle_{i}, z_{i}\right)\right\}
$$

and so are dist inct.

Claim. There are at least $v$ nonisomorphic triple systems among these at least $q !(q-1) ! \cdots 2 \cdot 1$ distinct associated triple systems.

This follows from the fact that each of these triple systems is based on the same $q v-1$ elements, and since there can be at most $(q v-1)$ ! triple systems in any one isomorphism class there are at least

$$
(q !(q-1) ! \cdots 2 \cdot 1) /(q v-1) !>v
$$

isomorphism classes. Hence regardless of the 3-skeins associated with the blocks other than $\left\{i, x_{i}, y_{i}, z_{i}\right\}$ there exist $v$ 3-skeins, say $\left(Q,\langle,\rangle_{i 1}\right)$ $, \cdots,\left(Q,\langle,,\rangle_{i v}\right)$, such that if $\left(Q \times V, b_{1}^{*}\right), \ldots,\left(Q \times V, b_{v}^{*}\right)$ are any $v$ quadruple systems obtained by successively associating $\left(Q,\langle,\rangle_{i 1}\right)$, $\cdots,\left(Q,\langle,,\rangle_{i v}\right)$ with the block $\left\{i, x_{i}, y_{i}, z_{i}\right\}$, then the $v$ triple systems obtained by deleting $(1, i)$ from each of these quadruple systems are nonisomorphic. Hence it is possible to choose $v$-skeins $\left(Q,\langle,,\rangle_{i}\right), i=1,2$, $\cdots, v$, such that if $\left(Q \times V, b^{*}\right)$ is any quadruple system obtained by associating $\left(Q,\langle,\rangle_{i}\right)$ with the block $\left\{i, x_{i}, y_{i}, z_{i}\right\}, i=1,2, \cdots, v$, then 
the $v$ Steiner triple systems obtained from $\left(Q \times V, b^{*}\right)$ by deleting $(1,1)$, $(1,2), \cdots,(1, v)$ are nonisomorphic. This completes the proof.

Corollary 4. Let $t$ be any positive integer. Then there is a Steiner quadruple system having at least $t$ NATS.

Proof. Choose $q$ and $v$ satisfying the conditions of Theorem 3 with the additional proviso that $v \geq t$.

4. Problem. As mentioned in the Introduction, prior to the results in this paper, the maximum number of NATS obtained from any quadruple system was 2 [4], [6]. Although the results in this paper rectify this situation, the size of the quadruple system having $t$ NATS is large compared to $t$. For example, taking $t=v=8$ and $q=50$ gives a quadruple system of order 400 having at least 8 NATS. A construction in which the size of the quadruple system having $t$ NATS is close to $t$ would be desirable. In particular, are there any quadruple systems of order $n$ having $n$ NATS (the best possible result along these lines)?

\section{REFERENCES}

1. Marshall Hall, Jr., Distinct representatives of subsets, Bull. Amer. Math. Soc. 54 (1948), 922-926. MR 10, 238.

2. P. Hall, On representatives of subsets, J. London Math. Soc. 10 (1935), 26-32.

3. H. Hanani, On quadruple systems, Canad. J. Math. 12 (1960), 145-147. MR 22 \#2558.

4. C. C. Lindner, Some remarks on the Steiner triple systems associated with Steiner quadruple systems, Colloq. Math. (to appear).

5. - On the construction of nonisomorphic Steiner quadruple systems, Colloq. Math. 29 (1974), 303-306.

6. N. S. Mendelsohn and S. H. Y. Hung, On the Steiner systems $S(3,4,14)$ and $S(4,5,15)$, Utilitas Math. 1 (1972), 5-95. 